\title{
DUST FORMATION AROUND WC STARS
}

\author{
P.M. WILLIAMS \\ Royal Observatories, Edinburgh, U.K.
}

\begin{abstract}
The presence of heated circumstellar dust around WC type Wolf-Rayet stars is reviewed in terms of the episodic or persistent condensation of carbon grains in their stellar winds. At present, 19 WC stars are known to form dust persistently and 7 episodically. Infrared spectroscopy suggests that the grains are amorphous rather than graphitic carbon. The properties of the stars which make dust episodically are reviewed in terms of processes in colliding winds in WR binary systems and the possible applicability of these processes to the stars which make dust persistently in examined.
\end{abstract}

Key words: stars: WC - individual: WR140 - circumstellar grains

\section{Introduction - the incidence of circumstellar grains}

It has long been evident from their infrared emission that carbon grains are present in the winds of many WC type WR stars and that this is a strong function of spectral subtype (e.g., Allen, Harvey \& Swings 1972; Gehrz \& Hackwell 1974, Cohen, Barlow \& Kuhi 1975 and Williams, van der Hucht \& Thé $1987 \mathrm{a}=$ WHT). The significance of this emission stems from the difficulty of the survival (let alone formation!) of grains near such hot stars. By considering the radiative equilibrium on a dust grain of known properties, we can determine the minimum distance from the star at which it can survive without sublimation. This is a few hundred AU and, at this distance, the WR stellar wind density $\left(\sim 10^{-17} \mathrm{~g} \mathrm{~cm}^{-3}\right.$ for a uniform wind) seems too low to allow grain formation or growth but is still high enough for any grains to be momentum-coupled to the wind (WHT). Consequently, dust grains are carried away from the star by the wind and cool as the stellar radiation heating them is further diluted, causing their emission to fade. Any dust made in an earlier evolutionary phase would long have been swept away. The observation that the infrared emission from some of the WC stars is persistent demonstrates that the cooling grains being dispersed are continually being replaced by the condensation of new grains in the wind. If this process occurs for only part of the time in a particular star and grain replenishment ceases, the infrared spectrum will show the effect of grain cooling and the emission will fade. This is the signature of the 'episodic' dust makers.

Our present knowledge of the incidence of heated circumstellar grains amongst galactic WC stars is summarized as a function of spectral subtype in Table I. Stars are identified by their WR numbers from the 6th Catalogue (van der Hucht et al. 1981) or subsequent interpolations in it: WR48a (Danks et al. 1983) and WR98a = IRAS 17380-3031 (Cohen et al. 1991). Radiation 
TABLE I

Incidence of heated dust as a function of subtype

\begin{tabular}{cccc}
\hline Type & Persistent & Episodic & None (?) \\
\hline WC9 & WR59 65 6973 & WR70 & WR81 88 92 \\
& $\begin{array}{c}76809596 \\
103104106112\end{array}$ & $98 \mathrm{a}$ & \\
& 117118119121 & & \\
\hline WC8 & 53113 & $48 \mathrm{a}$ & 116077 \\
& & & 101135 \\
\hline WC7 & none & 125 & 425056 \\
& & 137 & 57646879 \\
& & 140 & 869093 \\
\hline WC4 & none & 19 & incomplete \\
\hline
\end{tabular}

from heated grains has been observed from 18 of the 21 WC9 stars, 4 of the 9 WC 8 stars, 3 of the 13 WC7 stars and one WC4 star. Omitted from the table for reasons of space are the many WC5-6 stars which have been observed in one or more of the surveys cited or by van der Hucht et al. (in preparation) and none of which has shown dust emission. As each of the WC7 and WC4 type episodic dust makers is observed to be free of grain emission for some of the time, it is possible that more such systems remain to be discovered amongst the stars in the 'None (?)' column by occasional re-observation over a period of years.

The emission from the persistent dust makers appears to be constant, within the limitations of isolated observations made in different photometric systems, indicating constant grain replenishment. Two WC9 stars, WR70 and WR98a, show variable dust emission, perhaps attributable to episodic topping up of reservoirs of dust comparable to those of the persistent dust makers, and are included in the 'Episodic' column.

\section{Properties of the dust clouds and grains}

We still have direct size information for only one of the dust clouds. Allen, Barton \& Wallace (1981) and Dyck et al. (1984) made speckle observations of WR104 (Ve2-45), deriving N-S uniform disk angular diameters $\alpha_{U D}=0^{\prime \prime} .13$ at $2.2 \mu \mathrm{m}, 0^{\prime \prime} .25$ at $3.8 \mu \mathrm{m}$ and $0^{\prime \prime} .31$ at $4.8 \mu \mathrm{m}$. These are consistent with a circumstellar cloud in which the grain temperature falls off with increasing distance from the star and WHT's model for this system. 
The luminosity of each star's dust emission is readily determined from the infrared photometry and is relatively insensitive to reddening. In most cases, the ratio of the luminosity of the dust cloud to that of the stellar system (which is sensitive to reddening) is less than 0.1 but there are a few cases (WR76, WR104, WR118 and WR48a at maximum) where this ratio exceeds 0.5 (WHT). The dust-to-stellar luminosity ratios are the fractions of stellar UV-visual luminosity absorbed and re-radiated by the dust and, if the dust were distributed about the stars isotropically, would give the UV-visual optical depths directly. This should provide little circumstellar extinction. However, in the case of WR104, Cohen et al. (1975) found, from analysis of the interstellar $\mathrm{NaD}$ lines, that only part of the extinction observed towards this star was interstellar and that there was a significant circumstellar component. Cohen \& Kuhi (1977) derived $A_{V} \sim 4.7$ for the latter, suggesting that we view the system edge-on through a flattened dust distribution. Danks et al. suggested that WR48a had a significant circumstellar component to its extinction but Williams, van der Hucht \& Thé (1987b) found that the strength of the diffuse $\lambda 6284$ interstellar feature was consistent with the observed reddening being wholly interstellar. Because the dust emission from this star varies, its reddening should be observed periodically to see if it correlates with the dust emission.

Analysis of the infrared spectral energy distributions (SEDs) allows determination of the grain temperature range and emissivity law. Early studies by Allen et al. (1972), Gehrz \& Hackwell (1974) and Cohen et al. (1975) favoured graphitic grains but WHT preferred amorphous carbon on account of a better fit to the the SED of WR140 during its 1985 grain formation episode. The difference in emissivity laws between graphite $\left(\kappa \propto \lambda^{-2}\right)$ and amorphous carbon $\left(\kappa \propto \lambda^{-1}\right)$ is small and attempts to distinguish between them using observed SEDs is dependent on the temperature distribution of the grains. A less model-dependent diagnostic is the $11.52-\mu \mathrm{m}$ graphite resonance predicted by Draine (1984). Glasse et al. (1986) did not find it in the spectrum of WR104. The spectrum of WR140 taking during outburst in 1993 does not show it either (Fig. 1). These two stars may be considered as the archetypal persistent and episodic dust-makers respectively and, given the differences between them and their dust clouds and the absence of the $11.52-\mu \mathrm{m}$ resonance from both, it is most unlikely that the dust forming in WC stellar winds is graphite.

So far, the only spectroscopic feature known in WR dust emission is a broad one near $7.7 \mu \mathrm{m}$ observed in airborne and IRAS spectra of WR104 and WR112 and attributed to a carbonaceous carrier with small aromatic domains - either PAH molecules or amorphous carbon grains (Cohen, Tielens \& Bregman 1989). The $7.7 \mu \mathrm{m}$ feature gives IRAS LRS spectra of dustmaking WC stars a characteristic shape which led to the identification of IRAS 17380-3031, which shared this shape, as a WC star (Cohen et al. 1991 


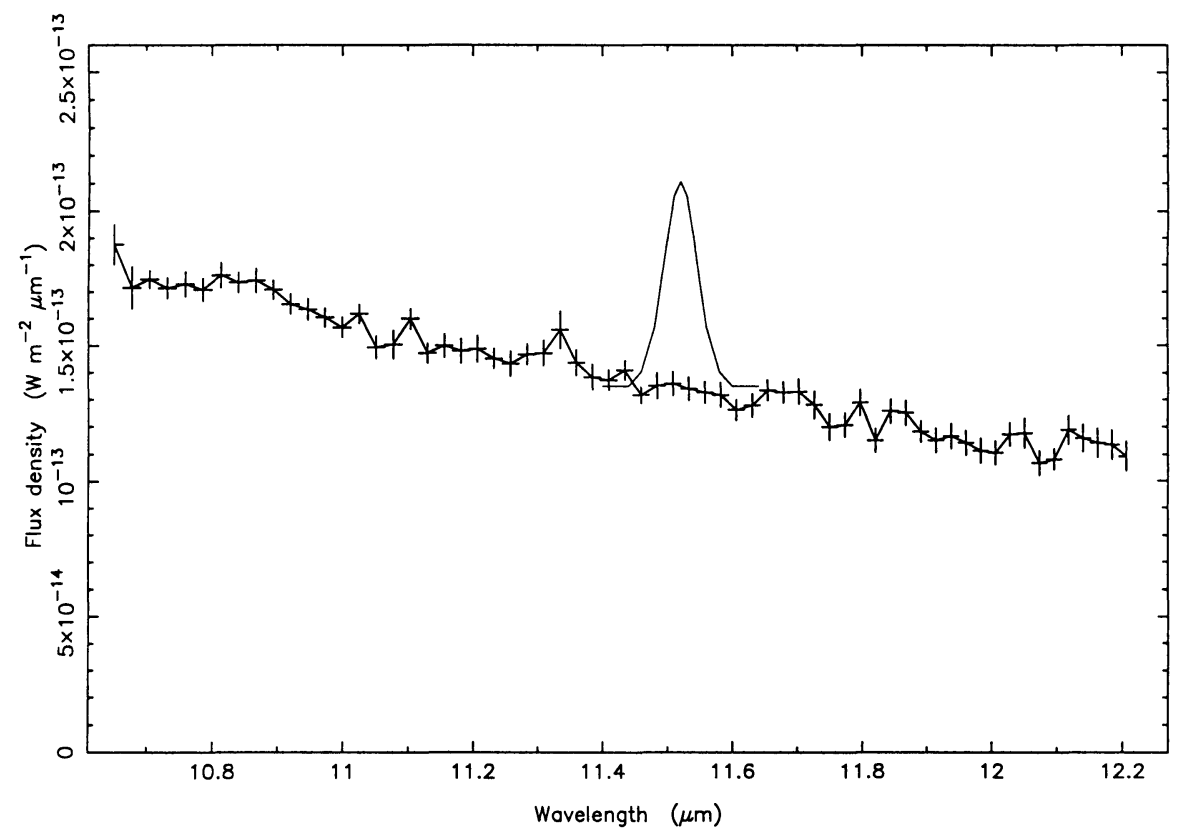

Fig. 1. Spectrum of WR140 at resolution $\Delta \lambda=0.06 \mu \mathrm{m}$ observed with UKIRT in 1993. Superimposed on the spectrum is a Gaussian profile at the position of the graphite resonance, convolved with the instrumental profile and having equivalent width $W_{\lambda}=0.05 \mu \mathrm{m}$ to show the feature expected if the dust comprised small graphite grains.

and these proceedings.). The absence of the common 11.3- $\mu \mathrm{m}$ PAH feature from the spectrum of WR140 (Fig. 1) and the low resolution spectra of persistent dust-makers observed by Roche \& Aitken (1984) is consistent with the absence of hydrogen in WC stellar winds.

Also not observed are features from likely molecular or atomic precursors to carbon grains, such as $\mathrm{C}_{2}$ or $\mathrm{C}_{\mathrm{I}}$ - not even $\mathrm{C}_{1}$ multiplet no. 1 seen in emission in young novae at $1.0695 \mu \mathrm{m}$. This puts severe limits on the abundances or, in the case of absorption lines, the location of these species.

\section{The episodic dust makers}

Since the Bali symposium (IAU 143), dust formation epsiodes have been observed from WR140 (HD 193793) and WR125 (IC14-36). That by WR140 had been predicted (e.g., Williams et al. 1987b) and was one of the drivers for this Symposium. Dust formation by WR125 was observed during infrared monitoring driven by the manifest similarity of this system to WR140 


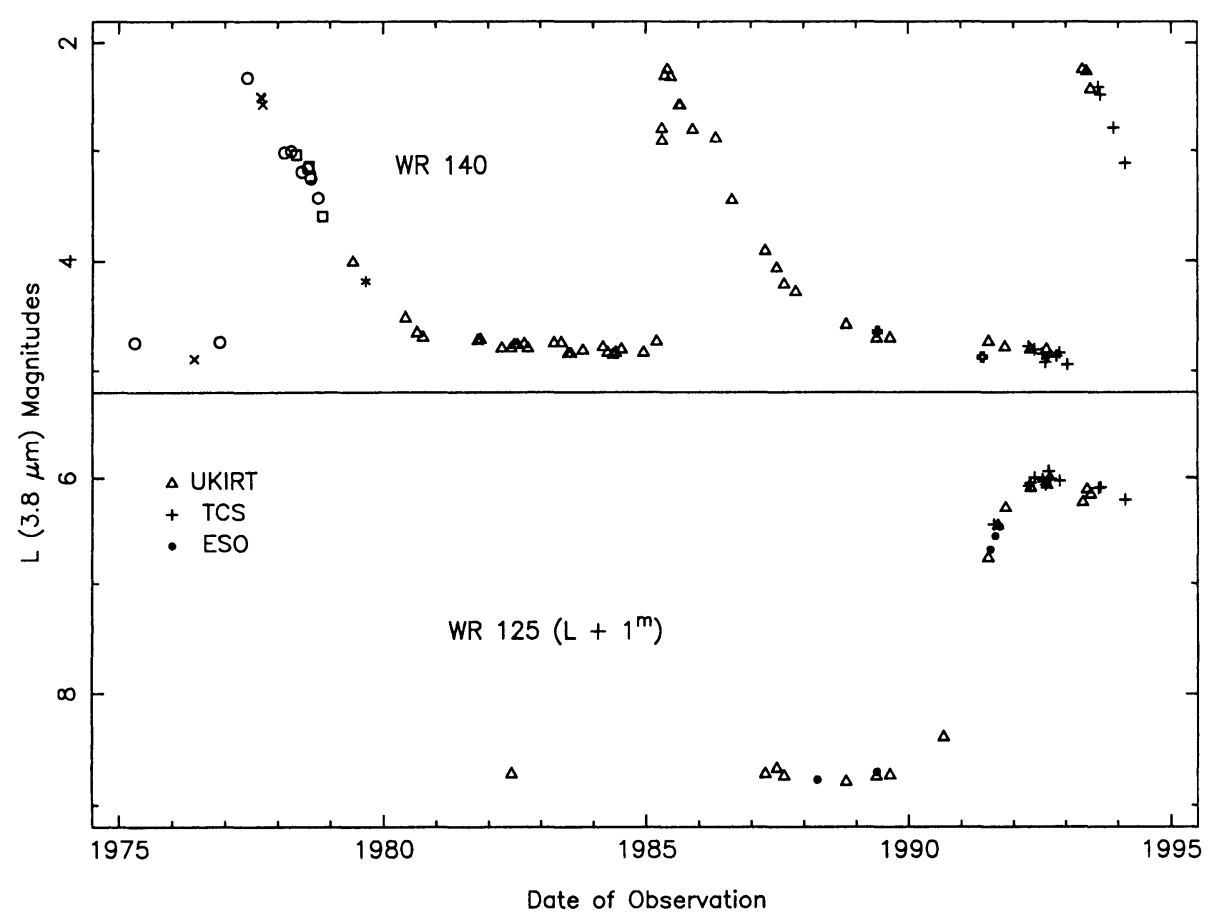

Fig. 2. $L(3.8 \mu \mathrm{m})$ photometry of WR140 and WR125. Different symbols mark observations made at different observatories.

spectroscopically and in its X-ray and radio behaviour (Williams et al. 1992). Synoptic infrared photometry of these two stars is plotted in Fig. 2. Observations of the 1993.3 episode by WR 140 confirmed the $2900 \mathrm{~d}$ period found by Williams et al. $(1990=$ W90 $)$ but were unable to define the rapid rise because WR140 was most unfavourably placed for observing at the time. Light-curves at three wavelengths, $1.65 \mu \mathrm{m}(H), 3.8 \mu \mathrm{m}$ and $8.75 \mu \mathrm{m}$ phased to the $2900 \mathrm{~d}$ period are compared in Fig. 3. Apart from the sharp rises at all three wavelengths, the light-curves are very different. That at $1.65 \mu \mathrm{m}$ shows the fastest decline, returning to quiescence around phase 0.2 , whereas it is not obvious that the $8.75 \mu \mathrm{m}$ flux has stopped fading before the next outburst occurs. The long-wavelength maximum is very much broader, with peak flux observed a year after that at shorter wavelengths. The behaviour at $3.8 \mu \mathrm{m}$ is intermediate; there is a hint of a shoulder on the fading light-curve a year after maximum and the flux falls to its wind level shortly before the next outburst. Models fitted to the 1985 observations by W90 indicated that $2.8 \times 10^{-8} M_{\odot}$ of amorphous carbon condensed between 1985.21 and 1985.54, forming an expanding cloud. The rate of dust formation, $\sim 8 \times 10^{-8} \mathrm{M}_{\odot} \mathrm{y}^{-1}$, 


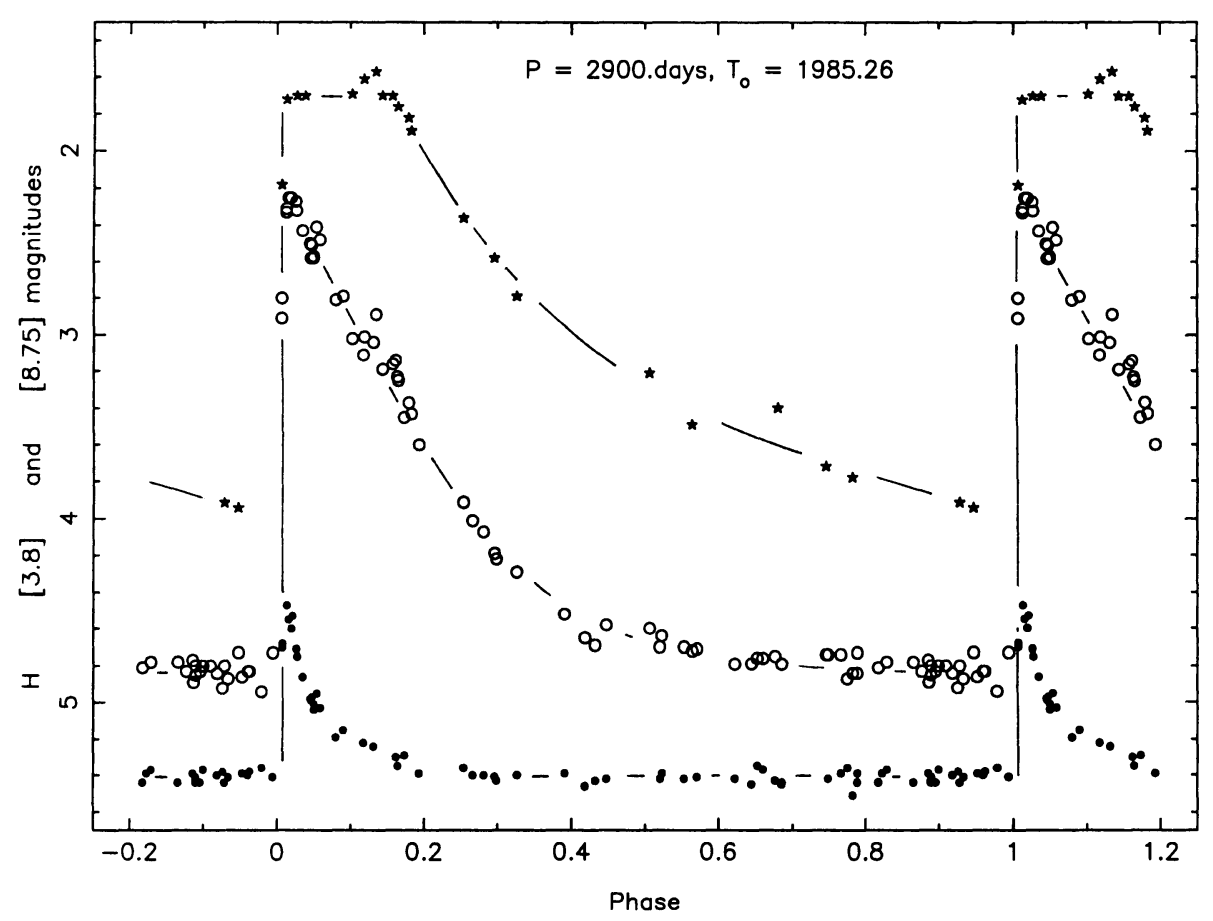

Fig. 3. Photometry of WR140 at $8.75 \mu m(\star), 3.8 \mu m(\circ)$ and $H(1.65 \mu m, \bullet)$ phased to the photometric period and time of periastron passsage in W90.

corresponds to $0.13 \%$ of the mass loss $\left(\sim 6 \times 10^{-5} \mathrm{M}_{\odot} \mathrm{y}^{-1}\right)$ or $\sim 5 \%$ of the total carbon being lost by the WC7 star.

The significance of WR140 is that the episodes are periodic and that the WC star is a member of a binary system with the same period. The eccentricity of the binary orbit was found to be very high and dust formation to occur during periastron passage when interaction of the WC7 and 04-5 stellar winds was at its greatest (W90). The conditions under which the colliding winds in the interaction region can be compressed and cool sufficiently to allow dust condensation have been discussed by Usov (1991). Thus WR140 provides a valuable clue to the achievement of conditions, notably enhanced density, allowing grain formation in WC stellar winds.

Observations of WR125 during 1991-4 (Fig. 2) show its dust formation was much more prolonged than that by WR140, continuing for about two years until late 1992. We should distinguish the three time-scales of flux rising, which depends on how long conditions in the wind allow dust condensation to continue, flux fading, which depends on the wind velocity, and recurrence, which is unrelated to the wind but, by analogy with WR140, 
TABLE II

The WC4-8 episodic dust-makers

\begin{tabular}{cccccc}
\hline & $\begin{array}{c}\text { WR140 } \\
\text { HD 193793 }\end{array}$ & $\begin{array}{c}\text { WR125 } \\
\text { IC14-36 }\end{array}$ & $\begin{array}{c}\text { WR137 } \\
\text { HD 192641 }\end{array}$ & WR48a & WR19 \\
sp. type & WC7+O4-5 & WC7+O9 & WC7+OB & WC8 & WC4 \\
\hline $\begin{array}{c}\text { maxima } \\
\text { at }\end{array}$ & 1977.4 & 1992.7 & 1984.5 & 1979.5 & $(1987 ?)$ \\
$3.8 \mu \mathrm{m}$ & 1985.4 & & & & \\
\hline$t_{\text {rise }}$ & 1993.3 & & & & \\
$t_{\text {fade }}$ & $5.3 \mathrm{y}$ & $2.1 \mathrm{y}$ & $\sim 3 \mathrm{y}$ & $>1 \mathrm{y}$ & $?$ \\
$t_{\text {recur }}$ & $7.94 \mathrm{y}$ & $>15 \mathrm{y}$ & $\sim 13 \mathrm{y}$ & $>15 \mathrm{y}$ & $>6 \mathrm{y}$ \\
\hline
\end{tabular}

is the period of the hypothetical binary assumed to trigger the episodes. Because the terminal velocity of the WC7 stellar wind and amplitude of infrared variation of WR125 are comparable to those of WR140, we expect its dust fading time to be comparable too, e.g., $4-5$ years at $3.8 \mu \mathrm{m}$. Together with the $\sim 2.1 y$ rise-time, this suggests that we could not have missed an outburst between the 1982 and 1987 observations and that any previous maximum must have occurred some time before 1978. Accordingly, if outbursts by this star are recurrent, the period must be greater than $15 y$. It has not yet been demonstrated that WR125 is a binary; the spectrum (O9) of the companion to the WC7 star has only recently been classified (Williams et al. 1994) and radial velocity observations over many years will be required to search for and establish an orbit.

The third episodic dust maker containing a WC7 star is WR137 (HD 192641), which rose to maximum in 1984.5 (Williams et al. 1985). Its fading has been slower than that of WR140, mostly because its wind velocity is only two-thirds that of WR140, and there are irregularities in the decline suggesting further minor bursts of dust formation (Williams \& van der Hucht $1994=\mathrm{WH})$. Comparison of the fading after 1984 with earlier observations in 1973-5 suggests a recurrence time of $\sim 13$ year for this system so it will be important to observe it frequently in the years leading up to the maximum expected in about 1997. Although this star has a composite spectrum, its status as a binary is uncertain. Annuk (1991) has determined an orbit from photographic spectra, but Underhill (1992) did not find systematic motion in spectra observed between 1986.6 and 1991.6. Like WR140, this is a relatively bright star, and it is to be hoped that the questions of the orbit and recurrence of dust formation can be solved in the next five years when we expect its activity to be greatest. 


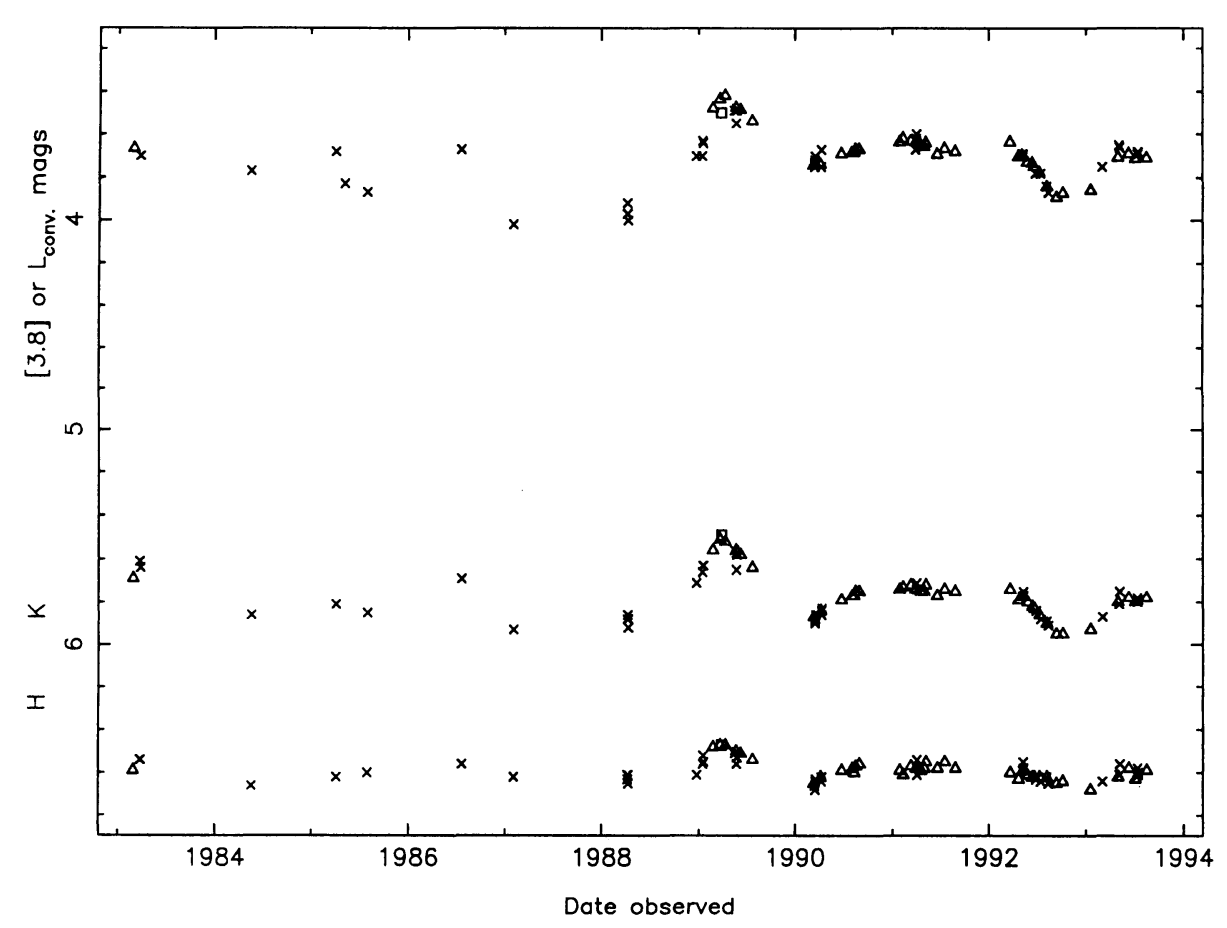

Fig. 4. Photometry of WR70 at $3.8 \mu m(x)$ or $L$ (converted to $3.8 \mu \mathrm{m}$ magnitudes using an empirical colour equation, $\Delta), K$ and $H$.

The fourth WC star for which a well defined dust formation episode has been observed is WR48a, discovered by Danks et al. (1983) on the rise to its maximum in 1979.5. The fading of its emission has been observed since 1982 and is the slowest of the stars yet observed (WH), perhaps attributable to a very low terminal velocity. Heavy reddening and proximity to an optically brighter star make this a difficult system for spectroscopy and we do not know whether it has a companion.

The observation in 1988 that the early (WC4) type star WR19 had a circumstellar dust shell, which faded over the next two years (Williams et al. 1990b), was unexpected given the early subtype of the star. The dilution of the emission lines in its spectrum suggests that it has a companion $0^{m} .8$ brighter than the WC4 star in the visible (Smith et al. 1990). The spectroscopy should be extended to the violet, where there is the best chance of observing absorption lines from the companion if it is a hot star, and a search for orbital motion undertaken.

Some of the properties of these five stars are collected in Table II, where the times refer to the flux at $3.8 \mu \mathrm{m}$. Only for WR140 do we have a reason- 
ably secure period and orbit. The others vary on long time-scales and it will take observations over at least another decade to determine the extent to which they resemble WR140 and contribute to our understanding of grain formation.

The WC9+B0 binary WR70 (HD 137603) varies on a shorter time-scale and in more complex way than the stars discussed so far. It showed a well defined rise to maximum in 1989.25 attributable to an increase in the amount of heated dust but the subsequent behaviour is harder to understand (Fig. 4). A spectroscopic study of WR70 by Golombek (1983) found velocity variations from both the WC9 and B0 stars but no satisfactory period. A plot of the B0 star velocity against time suggests a slow variations on time-scale of years ( $c f$. also Niemela, these proceedings). There is an urgent need for an orbit and more infrared observations to see how these relate.

\section{The persistent dust makers}

To form grains in WC stellar winds, we need higher densities than found in uniform, spherical winds $\left(\sim 10^{-17} \mathrm{~g} \mathrm{~cm}^{-3}\right.$ at the grain condensation radii, WHT), although grain growth by ion accretion may be facilitated by the grains' drift velocity relative to the wind (Zubko, Marchenko \& Nugis 1992). The candidate conditions for producing higher density regions are clumping, a disk structure for the wind and compression in a colliding wind binary. The last is exemplified by the periodic dust formation by WR140 when the wind collision region moves to a higher density region of the WC stellar wind during periastron passage. It seems reasonable to suggest that an identical pair of stars in a circular orbit whose separation was equal to that of WR140 at periastron would be able to make dust all the time and so appear as a persistent dust-maker. It is tempting to invert this and suggest that persistent dust-makers are colliding wind binaries (CWBs) whose separations fall in some favourable range (for the winds and luminosities of both stars) for grain formation like WR140.

A necessary condition is that persistent dust-makers are CWBs. There is evidence from dilution of emission lines that some of the persistent dustmakers have luminous companions (e.g., Cohen \& Kuhi 1977) but there is no evidence for a luminous companion to the WC9 star in WR103 (Smith \& Aller 1971) - whatever else makes up this system - so this dust-maker, at least, is not a CWB. More work on the spectra of the diluted emission line WC9 stars is needed to confirm that they are binaries.

We still don't know how grains form in WC stellar winds. The conditions are very different from the cool evolved stars which do this and the search for these conditions will lead to a better understanding of structures in the winds. 


\section{Acknowledgements}

The spectrum in Fig. 1 was taken in the UKIRT Service Observing programme, as was much of the photometry in Figs. 2 and 3. Other unpublished photometry in Figs 2-4 comes from the IAC, SAAO, ESO and SPM for which I thank Mark Kidger, Patricia Whitelock, Patrice Bouchet, Diah Setia Gunawan and Mauricio Tapia. I am also grateful to Dan Golombek for a copy of his thesis.

\section{References}

Allen, D.A., Harvey, P.M., Swings, J.P. 1972, $A \mathscr{E A} 20,333$

Allen, D.A., Barton, J.R., Wallace, P.T. 1981, MNRAS 196, 797

Annuk, K. 1991, in: K.A. van der Hucht \& B. Hidayat, B., Wolf-Rayet Stars and Interrelations with Other Massive Stars in Galaxies, Proc. IAU Symp. No. 143 (Dordrecht: Kluwer), p. 245

Cohen, M., Barlow, M.J., Kuhi, L.V. 1975, A\&A 40, 291

Cohen, M., Kuhi, L.V. 1977, MNRAS 180, 37

Cohen, M., Tielens, A.G.G.M., Bregman, J.D. 1989, ApJ (Letters) 344, L13

Cohen, M., van der Hucht, K.A., Williams, P.M., Thé, P.S. 1991, ApJ 378, 302

Danks, A.C., Dennefeld, M., Wamsteker, W.M., Shaver, P.A. 1983, $A \& A$ 118, 301

Draine, B.T. 1984, ApJ (Letters) 277, L71

Dyck, H.M., Simon, T., Wolstencroft, R.D. 1984, ApJ 277, 675

Gehrz, R.D., Hackwell, J.A., 1974, ApJ 194, 619

Glasse, A.C.H., Towlson, W.A., Aitken, D.K., Roche, P.F. 1986, MNRAS 220, 185

Golombek, D.A. 1983, PhD thesis, University of Buenos Aires

van der Hucht, K.A., Conti, P.S., Lundström, I., Stenholm, B. 1981, Sp. Sci. Rev. 28,227

Roche, P.F., Aitken, D.K. 1984, MNRAS 208, 481

Smith, L.F., Aller, L.H. 1971, ApJ 164275

Smith, L.F., Shara, M.M., Moffat, A.F.J. 1990, $A p J$ 348, 471

Underhill, A.B. 1992, $A p J$ 398, 636

Usov, V.V. 1991, MNRAS 252, 49

Williams, P.M., Longmore, A.J., van der Hucht, K.A., Talavera, A., Wamsteker, W.M., Abbott, D.C., Telesco, C.M. 1985, MNRAS 215, 23P

Williams, P.M., van der Hucht, K.A., Thé, P.S. 1987a, $A \& A$ 182, 91 (WHT)

Williams, P.M., van der Hucht, K.A., Thé, P.S. 1987b, QJRAS 28, 248

Williams, P.M., van der Hucht, K.A., Pollock, A.M.T., Florkowski, D.R., van der Woerd, H., Wamsteker, W.M. 1990a, MNRAS 243, 662 (W90)

Williams, P.M., van der Hucht, K.A., Thé, P.S., Bouchet, P. 1990b, MNRAS 247, 18P

Williams, P.M., van der Hucht, K.A., Bouchet, P., Spoelstra, T.A.Th., Eenens, P.R.J., Geballe, T.R., Kidger, M.R., Churchwell, E.B. 1992, MNRAS 258, 461

Williams, P.M., van der Hucht, K.A., Kidger, M.R., Geballe, T.R., Bouchet, P. 1994, MNRAS 266, 247

Williams, P.M., van der Hucht, K.A. 1994, in: C. Sterken \& M.J.H. de Groot (eds), The impact of Long-term Monitoring on Variable-Star Research, NATO ASI Conf. Series C436 (Dordrecht, Kluwer), p. 85

Zubko, V.G., Marchenko, S.V., Nugis, T. 1992, A\&A Transactions, 3, 131 


\section{DISCUSSION:}

Moffat: 1. WR140 and WR125 show very broad lines for their types (WC7, width implies WC5). Is this related to extreme wind collision or dust formation?

2. WR103 was shown by Stenholm to exhibit 1 mag dips lasting 1 month indicating a possible very long period binary perhaps involving a disk. Does your IR data relate to this in any way? Williams: 1 . I guess the line broadening may be by turbulence where the winds collide.

2. Whenever we've observed WR103, it has had the same infrared emission level and we haven't observed it when it is in its faint state.

Cherepashchuk: Could you say something new about IR observations of CV Ser, in particular about the deep and strange minimum of light observed by Hjellming and Hiltner in 1968.

Williams: I observed an infrared eclipse in 1976 but subsequent observations have found it to be steady.

Seggewiss: I reported on the fading of WR140 in 1993 (Panov et al., this volume). I wonder if there are colleagues here who also did observe the star in 1993 in the visual spectral region? Schulte-Ladbeck: We have obtained spectrophotometry at the same time as spectropolarimetry of WR140, in the spectral region from $3200 \AA$ to $7600 \AA$ (cf. Schulte-Ladbeck et al., this volume).

Niemela: Would you expect to see differences in polarization values for those stars which have circumstellar dust?

Williams: If only a small amount of the stellar visual flux is intercepted by the dust then, even though the dust is probably asymmetrically distributed, the polarization would be too small to observe.

Magalhaes: For modelling purposes, one needs parameters such as grain sizes, but when one measures in the $\mathbb{R}$, we are obtaining information on dust mass mostly. I would like to hear your comments on the possibility of getting a handle on grain sizes from the light curves at different wavelengths, such as the ones you presented.

Williams: So far, I have not allowed for grain growth but believe that this may partly account for the broad maxima in the 9-micron and longer wavelength light curves.

Pollock: WR140 at periastron has a similar dimension to $\gamma$ Vel at apastron. Why do you think that $\gamma$ Vel does not form dust in such similar circumstances?

Williams: Suitable conditions for dust formation must depend on luminosities of the stars and wind velocities as well. 